\title{
Erratum to: Expression of a Clostridium perfringens genome-encoded putative $N$-acetylmuramoyl-L-alanine amidase as a potential antimicrobial to control the bacterium
}

Glenn E. Tillman • Mustafa Simmons •

Johnna K. Garrish · Bruce S. Seal

Published online: 20 February 2014

(C) Springer-Verlag Berlin Heidelberg (outside the USA) 2014

\section{Erratum to: Arch Microbiol}

DOI 10.1007/s00203-013-0916-4

In the original publication, the oligonucleotide primer sequences were published incorrectly under the heading "Materials and methods" and the subheading "Cloning of a putative peptidoglycan hydrolases gene". The correct sentence should read as "The forward primer sequence was 5'-GCA CTA CAT ATG AAG ATA GCA GTA AGG GGT GGA- $3^{\prime}$ and the reverse primer sequence was $5^{\prime}$-GTG GTG CTC GAG ATC TAA ACT TAT ATA TTT TGC TG- ${ }^{\prime}$ (restriction site underlined)."

The online version of the original article can be found under doi:10.1007/s00203-013-0916-4.

G. E. Tillman · M. Simmons · J. K. Garrish · B. S. Seal $(\bowtie)$ Poultry Microbiological Safety Research Unit, Richard B. Russell Agricultural Research Center, Agricultural Research Service, USDA, Athens, GA 30605, USA

e-mail: Bruce.Seal@ars.usda.gov

Present Address:

G. E. Tillman · M. Simmons

Outbreak Section of the Eastern Laboratory, Food Safety

and Inspection Service, Richard B. Russell Agricultural Research

Center, USDA, 950 College Station Road, Athens,

GA 30605, USA 